\title{
Re-Print: An Interesting Case of Disseminated Tuberculoma of Brain and Spinal Cord Type of Study: Case Report
}

Parameshwar Keerthi B H, Anil Kumar Sakalecha*, Varun S, Shivaprasad G Savagave

Department of Radio-Diagnosis, Sri Devaraj Urs Academy of Higher Education \& Research, Tamaka, Kolar, Karnataka.

*Corresponding Author: Anil Kumar Sakalecha, Department of Radio-Diagnosis, Sri Devaraj Urs Academy of Higher Education \& Research, Tamaka, Kolar, Karnataka.

\section{Received Date: 24 August 2021 | Accepted Date: 22 September 2021 | Published Date: 01 October 2021}

Citation: Parameshwar Keerthi B H, Anil K Sakalecha, Varun S, Shivaprasad G Savagave. (2021). Re-Print: An Interesting Case of Disseminated Tuberculoma of Brain and Spinal Cord Type of Study: Case Report. Biomedical Research and Clinical Reviews. 5(1); DOI: 10.31579/26929406/083

Copyright: (c) 2021 Anil Kumar Sakalecha, This is an open-access article distributed under the terms of the Creative Commons Attribution License, which permits unrestricted use, distribution, and reproduction in any medium, provided the original author and source are credited.

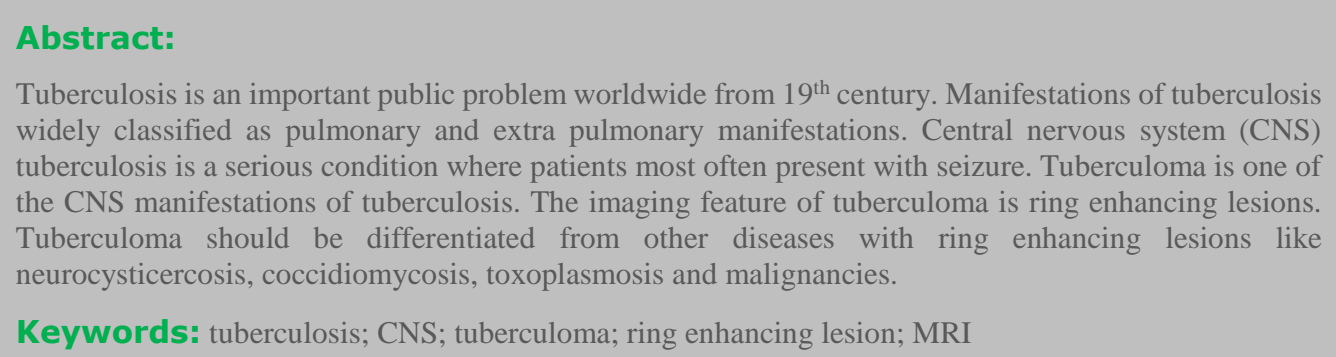

\section{Introduction:}

Tuberculoma of brain is an important manifestation of CNS tuberculosis. It constitutes approximately $5 \%$ of overall extra pulmonary manifestation of tuberculosis [1]. Spinal tuberculoma on other hand is extremely a rare manifestation occurring at the frequency of 1 in 50,000 individuals. It constitutes only $2 \%$ of CNS tuberculosis [2].

\section{Epidemiology:}

Tuberculosis (TB) is a great threat in developing countries with 8.6 million new cases and 1.3 million deaths worldwide in 2012. It is a disease of poverty affecting most of the underdeveloped and developing countires [3]. In India prevalence of tuberculosis is approximately 1.8 million cases per year [4].

\section{Case report:}

$30 \mathrm{Y} / \mathrm{M}$, a known case of tuberculosis on ATT for 3 months presented with history of weakness of both lower limbs. 1.5 Tesla MRI scanner
(SIEMENS® MAGNETOM AVANTO) was used for diagnosis.

Multifocal, well-defined, variable sized, rounded and ovoid, thick walled solid and cystic lesions with diffuse perilesional edema in bilateral cerebral and cerebellar hemispheres, predominantly at grey-white matter junctions, left pons, cerebellar vermis, cervical and thoracic segment of spinal cord at the level of C4 and D11 vertebral levels. Few of the lesions revealed internal T2 hyperintense signal-suggestive of liquefaction. Few of the lesions revealed central T2 hypointense signal- suggestive of caseation. On post-contrast study, the liquefactive lesions demonstrated smooth ring enhancement . Solid lesions demonstrated homogeneous enhancement. Diffuse cerebral edema noted in the form of effacement of sylvian fissure and cortical sulci. On MR-Spectroscopy elevated lipid peak (1.3 ppm), mildly reduced NAA (3.2 ppm), reduced NAA/Cho ratio were seen. All vertebrae were normal. I

Above imaging features were suggestive of disseminated central nervous tuberculoma. 

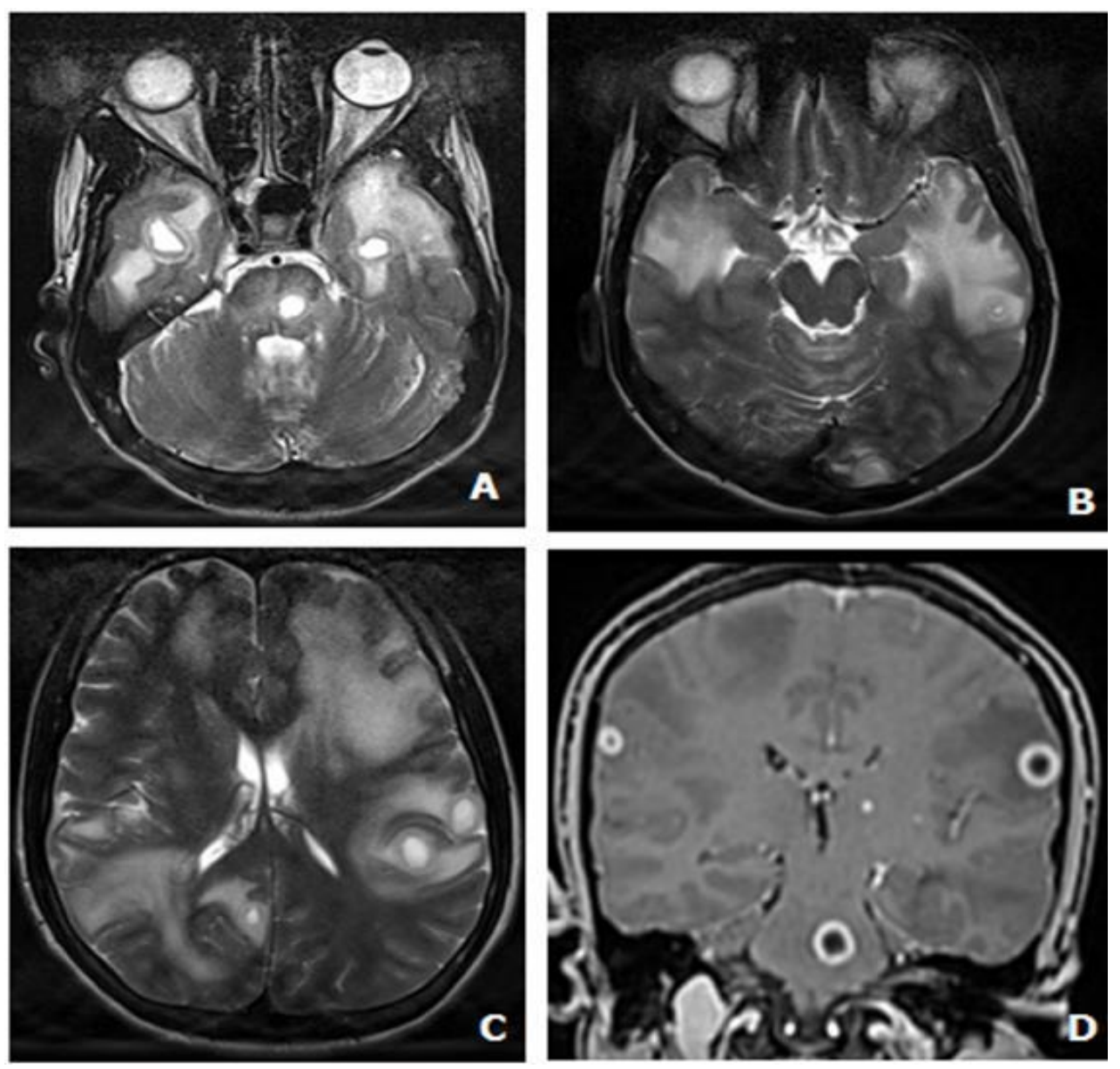

Figure 1: A-Multiple T2 hyperintese with peripheral hypointense liquefactive lesions in left pons and bilateral temporal lobes with moderate perilesional edema. B - T2 hypointense caeseating lesions in left occipital lobe and liquefactive lesion in left temporal lobe. C-Multifocal liquefactive lesions with moderate perilesional edema in left parietal and right occipital lobes. D - T1-FS Post contrast image showing smooth, thick ring ehancing lesions with moderate perilesional edema in left pons and bilateral parietal regions.
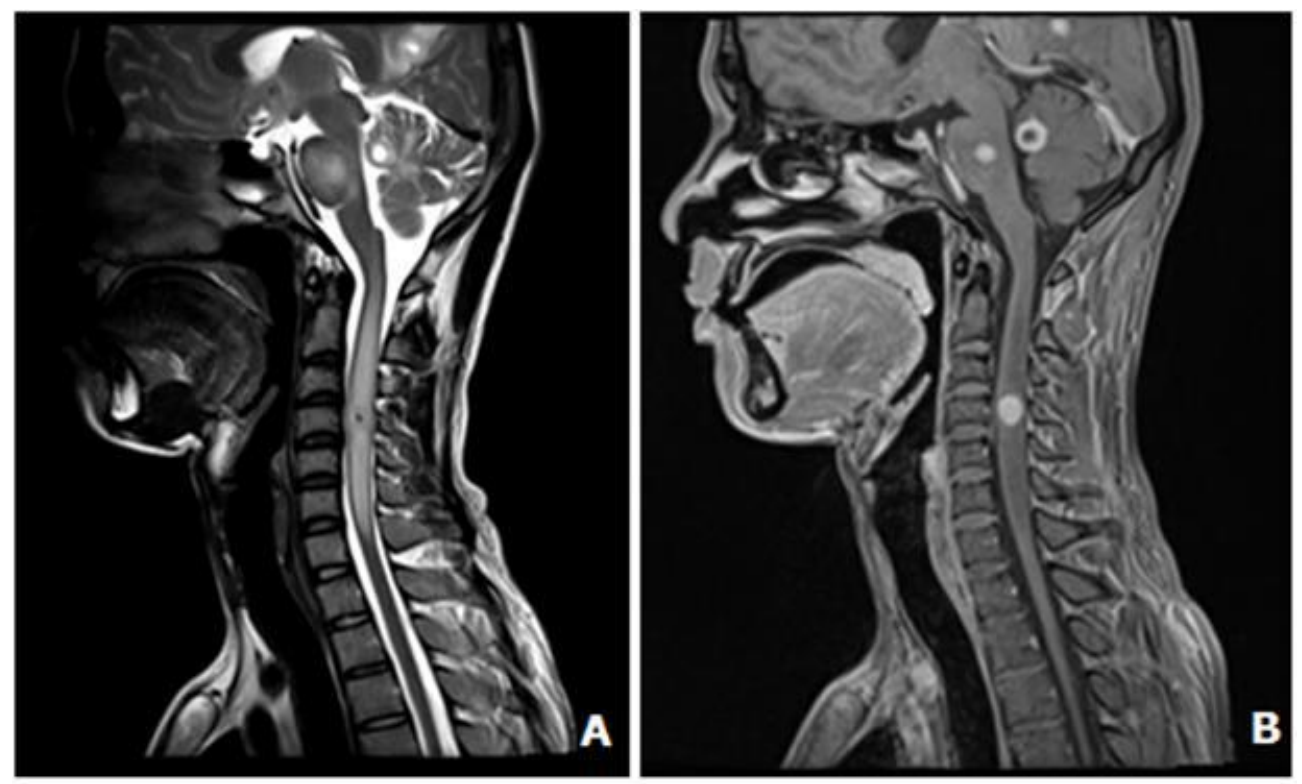

Figure 2: A T2 sagittal image of cervical spine showing hypointense caeseatig lesion with moderate perilesional edema in pons and spinal cord. Liquefactive lesions with mild perilesional edema in cerebellar hemisphere and occipital lobe. B - T1-FS post contarst image demonstartes homogeneous enhancement of spinal cord and pontine lesion and thick, smooth ring enhancement in cerebellar lesion. 


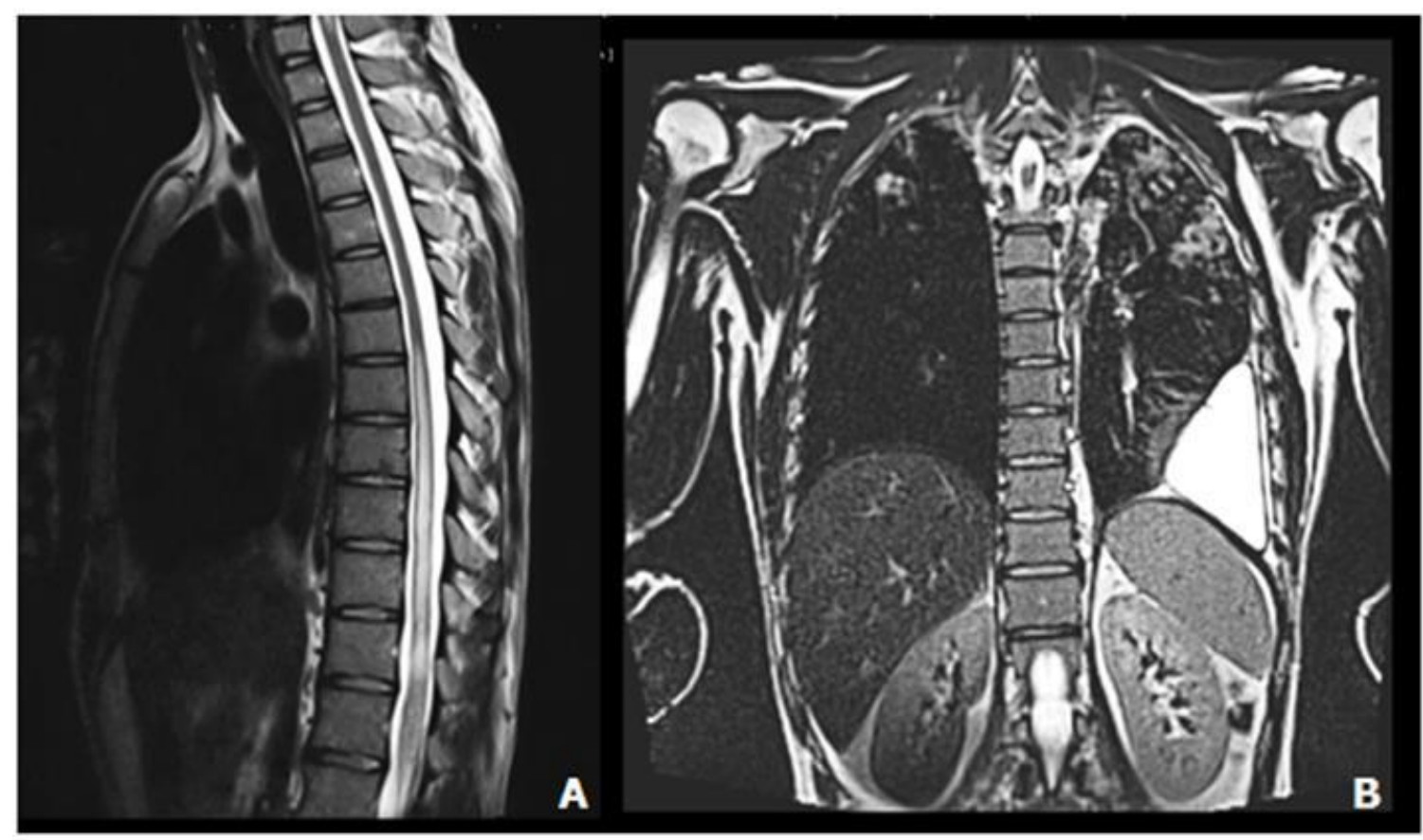

Figure 3: A T2 sagittal image of dorso-lumbar spine showing T2 hyperintense liquefactive lesion with moderate perilesional edema in the spinal cord. B - T2 coronal BLADE sequence of thorax showing left oculated pleural effusion with multiple 'tree-in-bud' appeareance in bilteral lung parenchyma.

\section{Discussion:}

Central nervous system (CNS) tuberculosis is a life threatening and devastating condition which is curable when diagnosed in early stages. Imaging manifestations of CNS tuberculosis are meningitis, tuberculoma, miliary tuberculosis, abscess, cerebritis, and encephalopathy. Tuberculoma is the most common parenchymal lesion in CNS tuberculosis which could be found in any portion of the intracranial space. The lesion may be solitary or multiple and may be seen with or without meningitis [5].

Most common clinical findings in CNS tuberculosis encountered is seizure followed by meningitis. Other manifestations include focal neurological deficits, behavioural changes, and altered sensorium [6].

Mode of spread for spinal tuberculoma is CSF spread, haematogenous spread or local spread from spinal tuberculosis. In our case spinal tuberculoma was associated with pulmonary tuberculosis. It is important to look for pulmonary tuberculosis in patients with spinal intramedullary tuberculomas. Lu $\mathrm{M}$ et al in his study also showed a positive association between spinal tuberculoma and pulmonary tuberculosis [7].

On MRI, the lesions are classified as non caseating granuloma, caseating granuloma, caseating granuloma with central liquefaction and calcified granuloma. Non caseating granuloma are iso-to-hypointense on T1, hyperintense on $\mathrm{T} 2$ weighted image with homogeneous enhancement on post contrast image. Caseating granuloma are hypointense on both $\mathrm{T} 1$ and T2 weighted image with hyperintense rim on T1 weighted image and homogeneous or ring enhancement on post contrast enhancement. Caseating granuloma with liquefaction are iso-to-hypointense lesion with peripheral hyperintense rim and hypointense with central hyperintensity on T2 weighted image with ring enhancement on post contrast image. Calcified granuloma are hypointense on $\mathrm{T} 1$ and $\mathrm{T} 2$ weighted image with no significant enhancement on post contrast study.

\section{Treatment:}

ATT treatment include isoniazid (INH) $300 \mathrm{mg} /$ day, rifampicin (RF) 450 $\mathrm{mg} /$ day, pyrazinamide $1500 \mathrm{mg} /$ day, and ethambutol $800 \mathrm{mg}$ /day daily for 2 months, followed by INH and RF for 4 months. Pyridoxine at 40 $\mathrm{mg} /$ day was given for all 6 months. Prednisolone at $1 \mathrm{mg} / \mathrm{kg}$ body weight was given for 1 month and then subsequently tapered over a period of 1 month. Follow up MRI imaging should be performed to look for the reduction in the size of the lesion. Pulmonary CT should also be performed to look for the regression of the lesions.

\section{References:}

1. Subhasis M, Runa D, Shabana B. (2015). Tuberculoma of the brain - A diagnostic dilemma: Magnetic resonance spectroscopy a new ray of hope. J Assoc Chest Phys. 3:3-8.

2. Thirunavukarasu S C, Ramachandrappa A. (2012) A rare case of intramedullary tuberculoma: Complete resolution after medical treatment and role of magnetic resonance imaging in diagnosis and follow-up. Asian J Neurosurg. 7:223-226.

3. Sulis G, Roggi A, Matteelli A, Raviglione M C. (2014) Tuberculosis: Epidemiology and Control. Mediterr J Hematol Infect Dis. 6:e2014070.

4. Sandhu G K. (2011). Tuberculosis: Current Situation, Challenges and Overview of its Control Programs in India. J Glob Infect Dis. 3:143-150.

5. Taheri M S, Karimi M A, Haghighatkhah H, Pourghorban R, Samadian M, Kasmaei H D. (2015). Central Nervous System Tuberculosis: An Imaging-Focused Review of a Re-emerging Disease. Radiol Res Pract. 202806.

6. Cherian A, Thomas S V. (2011). Central nervous system tuberculosis. Afr Health Sci. 11: 116-127.

7. Lu M. (2010). Imaging diagnosis of spinal intramedullary tuberculoma: case reports and literature review. J Spinal Cord Med. 33:159-162.

8. Khatri G D, Krishnan V, Antil N, Saigal G. (2018). Magnetic resonance imaging spectrum of intracranial tubercular lesions: one disease, many faces. Pol J Radiol.83:524-535. 
This work is licensed under Creative Commons Attribution 4.0 License

To Submit Your Article Click Here: Submit Manuscript

DOI: $10.31579 / 2692-9406 / 083$
Ready to submit your research? Choose Auctores and benefit from:

$>$ fast, convenient online submission

$>$ rigorous peer review by experienced research in your field

$>$ rapid publication on acceptance

$>$ authors retain copyrights

$>$ unique DOI for all articles

$>$ immediate, unrestricted online access

At Auctores, research is always in progress.

Learn more www.auctoresonline.org/journals/biomedical-research-andclinical-reviews- 